\title{
Do we (sufficiently) integrate practice into theory?
}

\author{
Thomas W. Guenther ${ }^{1}$ · Frank H. M. Verbeeten ${ }^{2}$
}

Accepted: 11 November 2021 / Published online: 25 November 2021

(c) The Author(s), under exclusive licence to Springer-Verlag GmbH Germany, part of Springer Nature 2021

Fairly recently, Shiva Rajgopal (Columbia Business School) argued that academic research in accounting has strayed from producing work that is useful to either practitioners or policy makers (Rajgopal, 2021). Rajgopal uses three criteria to arrive at that assessment: (1) the number of products and processes that accounting research has produced in the last 50 years, (2) the overlap between issues that Chief Financial Officers (CFOs) and Chief Executive Officers (CEOs) worry about and our published research, and (3) whether science or knowhow in academia in a particular area is ahead of that in practice. In addition, Rajgopal (2021) suggests several potentials reasons for the (perceived?) problem, and makes several suggestions on how to improve on the current situation.

While the objective of this editorial is not to repeat Rajgopal's (2021) analysis and arguments, some things stand out for our specific discipline, management accounting and control. First of all, Rajgopal indicates that we have not created many products, with the exception of Activity Based Costing and the Balanced Scorecard. These 'products' were developed through action research that may help to develop new solutions that alter existing practice, and test the feasibility and properties of this new solution. The Journal of Management Control is open to submissions of action research papers, and we will invite reviewers that are able to review such papers.

Another topic that is raised by Rajgopal is that our research may not deal with topics that are on the agenda of CFOs. For that purpose, Deloitte CFO Insights magazine is used as they publish a list of issues that keeps CFOs up at night. Some of the topics that are mentioned in this list are currently being picked up in research; for example, digitalization and big data issues in accounting departments appear to be on the rise if we look at conference presentations and journal submissions. However, other topics appear to attract less research attention. For example, while

Frank H. M. Verbeeten

f.h.m.verbeeten@uva.nl

Thomas W. Guenther

thomas.guenther@tu-dresden.de

1 Technische Universitaet Dresden, Dresden, Germany

2 University of Amsterdam, Amsterdam Business School, Amsterdam, The Netherlands 
digitalization will affect corporate structures, there is little research on how digitalization would affect the allocation of decision rights and the associated design of performance measurement and reward systems. Digitalization could have a number of effects: it can increase the data availability at top management level, which may facilitate centralization of decision rights. Alternatively, digitalization may provide better insights into the actions of lower level managers, providing the opportunity to better evaluate the performance of the lower level manager. Associated with the previous reasoning, performance measurement and reward systems are also likely to change; this provides substantial opportunities for research.

In addition, one of the issues mentioned by CFOs is innovation, both in terms of short term agility as well as in long term value creation. We have little knowledge what the demands are on finance to facilitate agility: is it a higher frequency in forecasting (continuous forecasting), participation in decentralized multi-disciplinary team structures, or other changes to the control system that may be required to achieve this? Our colleagues in operations management appear to be more practice-oriented, while still able to publish these results in recognized journals. Similar, in terms of long term value creation, current developments on Corporate Social Responsibility (CSR) and/or Environmental, Social and Governance (ESG) issues may be relevant for management accounting and control systems. For example, one of the papers in the current issue suggests that formal controls as well as informal controls (such as intrinsic motivation) may be key for implementing CSR activities. While this research area is not new, current developments in European legislation may provide new opportunities to address these issues. In addition to archival research, labotatory experiments and surveys, the Journal of Management Control is open to other research methods such as field studies, case studies and action research that help to advance our knowledge in the area of management accounting and management control. Relative to financial accounting, we may be at an advantage given that most business problems are managerial control questions, and we should be able to build upon that competitive advantage.

This fourth issue of the Journal of Management Control includes four very interesting research papers, covering topics which are relevant for academics and practitioners. Jennifer Kunz and Mathias Heitz provide a comprehensive literature review, focusing particularly on the embeddedness of risk culture into banks' management control systems. Various academic disciplines have discussed topics related to risk culture from varying methodological angles. While this provides a rich basis of theoretical and empirical evidence to guide business practice and improve regulation, the application of many approaches and methods can result in fragmentation and loss of a comprehensive perspective. The authors apply a systematic literature reviews (based upon 103 articles) and interpret the findings through the theoretical lens of management control research. In addition, the authors synthesize the findings in a comprehensive model and derive consequences for theory, business practice and regulation.

The second paper, by Markus Jung and Mischa Seiter, investigates why forecasters would show aversion to algorithms. Forecasts serve as the basis for many managerial decisions, including the allocation of funds and planning of activities. With the potential of new data sources and (big) data analysis techniques, management accountants and other forecasters are likely to interact with algorithms. Despite the fact that algorithms can show better forecasting performance than humans, forecasters may show aversion to the outcomes 
of algorithms. The authors investigate whether three environmental variables that have been identified in the management accounting literature (i.e., 'do your best' goals, time pressure and forecasters' data input decision rights) mitigate algorithm aversion. Based upon an experiment with 1840 participants, they find that time pressure mitigates algorithm aversion (due to a lack of confidence in their own forecast); the hypotheses for the other variables were not supported by the empirical results.

Kevin Baird and Vicki Baard examine the mediating role of team structural empowerment on the association between diferent aspects of management controls and team effectiveness (team performance and team viability). Specifically, they focus on management controls such as team structure (team standardisation and team discretion) and the use of controls (interactive and diagnostic use of controls). Using survey responses from 344 team members, they find that the associations between the management control system elements (team structure, use of controls) and team effectiveness is mediated by team structural empowerment. Hence, the authors recommend that managers leverage team structural empowerment to achieve greater team effectiveness; they can do this through the use of controls. In addition, their findings contribute to management accounting contingency literature by identifying the crucial role of the management control system for effective team work.

Finally, Leanne Johnstone explores the type and use of sustainability control in small to medium sized enterprises (SMEs) through the implementation of an environmental management system. The paper draws on the theoretical framework of sustainability control as an analytical tool to explore the interplay between the formal design of control instruments and the operational use of these in practice for the studied SMEs. Based upon a qualitative study of 18 SMEs and seven auditors operating in Northern Europe, the author indicates that both the formalised control instrument design and the operational use of these controls is important; however, environmental management in daily tasks is also achieved by engaging non-managerial employees through their passionate interests and intrinsic motivations. This suggests that a combination of formal controls and extra-organisational individual values and beliefs about sustainability. Particularly, engaging non-managerial employees in SMEs through a combination of extrinsic and intrinsic rewards appears valuable for a sustainable future.

We hope that the readers of the Journal of Management Control, academics as well as practitioners, may draw new insights from this issue. If so, the authors in this issue have contributed to the expansion of our knowledge in the field of management accounting and control, both for practice and research.

Amsterdam, December 2021

Frank Verbeeten and Thomas Guenther

Managing editors

\section{Reference}

Rajgopal, S. (2021). Integrating practice into accounting research. Management Science, 67(9), 5430-5454.

Publisher's Note Springer Nature remains neutral with regard to jurisdictional claims in published maps and institutional affiliations. 